\title{
Gases de átomos Fermiônicos aprisionados por uma rede quase periódica e um potencial harmônico bidimensional
}

\author{
Fermi Gas atoms trapped by one quasi-periodic lattice and a two-dimensional harmonic potential
}

\author{
Renan Paes ${ }^{1 @}$, Valdir A. do Nascimento², Elaine Silva de Padua Melo², Rita de Cássia Avellaneda \\ Guimarães $^{2}$, Valter Nascimento*2@

\footnotetext{
${ }^{1}$ Universidade Federal de Mato Grosso do Sul, Instituto de Física, Cidade Universitária, Campo Grande, MS, Brasil Campo Grande, MS, Brasil
} \\ ${ }^{2}$ Universidade Federal de Mato Grosso do Sul, Grupo de Espectroscopia e Bioinformática Aplicado a Biodiversidade e a Saúde,
}

Recebido em 03 de Abril, 2018. Revisado em 06 de Julho, 2018. Aceito em 18 de Julho, 2018

\begin{abstract}
Neste trabalho, nós consideramos um modelo tridimensional dinâmico de um gás de férmions em um estado superfluido de Bardeen-Cooper-Schrieffer aprisionado por uma rede óptica quase periódica unidimensional e na presença de um potencial externo em forma de charuto. Através da aproximação variacional, estudamos a possibilidade de que o coeficiente de não linearidade atuando em uma combinação com o potencial da rede óptica quase periódica e potencial em forma de charuto permite o surgimento de sólitons tridimensionais iluminados. As famílias de gaps sólitons fundamentais tridimensionais são estáveis para pequenos valores de amplitude da rede óptica quase periódica. O manuscrito apresentado pode tornar-se um guia de ensino e aprendizagem em estudos envolvendo gases fermiônicos aprisionados por laser. Os alunos são incentivados a realizar cálculos variacionais para a equação hidrodinâmica em uma dimensão.
\end{abstract}

Palavras-chave: Redes ópticas quase-periódicas, Superfluido de Fermi, Aproximação variacional (AV).

In this work, we consider a dynamical three-dimensional model of a Bardeen-Cooper-Schrieffer superfluid Fermi gas trapped in a unidimensional quasiperiodic optical lattice and in the presence of a tight cigar-shaped trapping potential. By variational approximation, we study the possibility that the nonlinearity coefficient acting in a combination with the potential of the quasiperiodic optical lattice and cigar-shaped trapping potential, allows three dimensional bright gap solitons to arise. Families of three-dimensional fundamental gap soliton are stable for small values of amplitude of the quasiperiodic optical lattice. The manuscript presented may become a teaching and learning guide in studies involving fermionic gases trapped by light fields. Students are encouraged to perform variational calculations for the hydrodynamic equation in one dimension.

Keywords: Quasiperiodic optical lattice, Fermi superfluid, variational approximation (VA).

\section{Introdução}

Proposta em 1925 a partir dos trabalhos teóricos de Albert Einstein e do físico indiano Satyendra Nath Bose, o condensado de Bose-Einstein trata-se de uma fase da matéria obtida quando átomos bosônicos estão a baixas temperaturas. Entretanto, a condensação de átomos bosônicos a baixas temperaturas somente foi comprovada experimentalmente em 1995, graças à utilização de lasers que criam potenciais de aprisionamentos que confinam os átomos [1]. Deste então, tem crescido o desejo de estudar as propriedades não lineares de fenômenos que envolvem átomos ultrafrios. Atualmente é possível confinar experimentalmente átomos fermiônicos utilizando lasers e criar potenciais adicionais como as redes ópticas periódicas $[2,3]$. Na presença de uma rede óptica, átomos são capturados nos máximos ou mínimos de intensidade de um campo de luz de ondas estacionárias devido às forças de

*Endereço de correspondência: aragao60@hotmail.com dipolos, permitindo aumentar a compreensão de vários fenômenos de muitos corpos; tal como a supercondutividade em alta temperatura, superfluidez e isolantes de Mott [4-6].

Os estudos teóricos da dinâmica de condensados são realizados através da equação de Gross-Pitaevskii, que possui como solução uma função de onda macroscópica que descreve o sistema de gases frios aprisionados. Tais funções de ondas possibilitam compreendermos experimentalmente as questões fundamentais de fenômenos que surgem em átomos frios e estão associados à física de estado sólido assim como às oscilações de Bloch e processos colisionais de sólitons [7-9]. Utilizando a estrutura periódica de uma rede óptica é possível controlarmos algumas propriedades de um condensado, como a estabilidade de excitações não lineares localizadas com potenciais químicos dentro das bandas [10, 11] e a formação de sólitons iluminados (denominados de gaps sólitons iluminados) [12]. É a partir do controle de uma rede óptica que cada 
sítio da rede passa a ser ocupado por apenas um átomo em seu estado fundamental; dessa forma, condensados revelam-se promissores como registros para computação quântica [13, 14].

Embora não possamos medir uma função de onda de um condensado, uma vez que a resolução óptica de um sistema de imagem não pode detectar átomos individuais, a distribuição do momento pode ser medida através do tempo de vôo dos átomos e interpretada teoricamente através de funções de ondas na forma gaussianas. Métodos variacionais têm obtido sucesso ao utilizar funções de ondas gaussianas como soluções da equação de GrossPitaevskii (1D, 2D e 3D) que representa gases bosônicos ou fermiônicos aprisionados por redes ópticas simples unidimensionais, bidimensionais ou tridimensionais. Métodos variacionais possuem a mesma precisão que alguns métodos numéricos $[15,16]$, e permitem estudar a estabilidade de gaps sólitons formados a partir das várias geometrias de aprisionamentos $[15,16]$.

Abordagens didáticas sobre a teoria de condensados de Bose-Einstein sem dar ênfase aos cálculos numéricos e considerando apenas potenciais harmônicos simples podem ser encontradas em alguns livros-texto de mecânica estatística e em artigos publicados em revistas de ensino [17-21]. Entretanto, durante alguns cursos de graduação em física, não existe uma cultura de ensinar as novas formas tecnológicas e teóricas de aprisionamentos de gases quânticos. Outra carência de ensino e aprendizagem que podemos destacar está voltada ao estudo da formação de sólitons, gaps e bandas de energias que surgem em gases quânticos aprisionados. De fato, existe uma carência em ensinarmos para os alunos de graduação ou até mesmo de pós-graduação dos cursos de ciências exatas, que é possível a formação de sólitons e gaps de energia em condensados de Bose-Einstein ou gases fermiônicos aprisionados por potenciais criados por lasers. Apesar de princípios variacionais e formalismo lagrangiano serem abordados na disciplina de mecânica clássica nos cursos de graduação de física ou engenharias, não existe aplicações de tais métodos variacionais voltados à solução da equação de Gross-Pitaevskii, que não deixa de ser uma equação de Schrödinger não linear e que descreve a dinâmica de gases quânticos aprisionados.

$\mathrm{Na}$ tentativa de suprir em partes algumas das necessidades citadas acima como a falta de livros didáticos ou outros materiais pedagógicos, alguns trabalhos têm sido publicados na Revista Brasileira de Ensino de Física, enfatizando a utilização de métodos variacionais e método de Monte Carlo aplicado ao oscilador harmônico unidimensional [22], ou destacando o uso do formalismo de supersimetria em mecânica quântica aliado ao método variacional [23]. Artigos com o propósito de apresentar a aplicação dos métodos variacionais como solução das equações hidrodinâmicas de campo médio tridimensional e unidimensional, demonstraram que é possível a formação de gaps sólitons iluminados em um gás de bósons ou em um gás de férmions degenerados confinados por um potencial harmônico e uma rede óptica unidimensional [24]. Por outro lado, estudos variacionais envolvendo átomos bosônicos aprisionados por redes simples ou duplamente periódicas unidimensionais, comprovaram analiticamente que é possível criar gaps sólitons iluminados sem a presença de um potencial harmônico [25]. Soma-se a esta seqüência de trabalhos publicados a solução da equação hidrodinâmica de campo médio que representa um gás de férmions unidimensional aprisionados por uma rede quase periódica, permitindo também a formação de sólitons iluminados [26]. Em decorrência de grandes esforços na busca de melhoria do processo de divulgação científica e de ensino, assim como da falta de abordagens didáticas envolvendo vários tipos de potenciais de redes ópticas, somos motivados a estudar a estabilidade espacial de átomos fermiônicos aprisionados por redes ópticas não periódicas, ou também denominadas de redes quase periódicas utilizando métodos variacionais.

Com o intuito de contribuirmos para a divulgação do ensino e aprendizagem, o presente trabalho apresenta uma abordagem utilizando a aproximação variacional como soluções de uma equação hidrodinâmica de campo médio em três dimensões que representa gases fermiônicos aprisionados por campos de luz. O objetivo foi estudar a formação de gaps sólitons iluminados em um gás de férmions degenerado confinados por uma rede óptica quase periódica unidimensional e na presença de um potencial transversal bidimensionais na forma de charuto. Através da aproximação variacional nós estudamos a possibilidade de que a não linearidade efetiva para férmions em um regime fraco atuando em combinação com o potencial de rede óptica quase periódica permitisse o surgimento de gaps sólitons fundamentais em três dimensões. Em face do exposto, o trabalho proposto é um complemento dos estudos citados nas referências [24-26] e propõe soluções variacionais para as equações hidrodinâmicas de campo médio tridimensional utilizando uma função gaussiana tridimensional.

Este manuscrito está organizado como segue. Na seção 2 faremos uma abordagem sobre sólitons e redes ópticas, destacando os conceitos físicos fundamentais envolvidos na formação de gaps sólitons fundamentais em condensados de Bose-Einstein. Na seção 3 nós apresentaremos a equação hidrodinâmica para Férmions ou equação de densidade funcional que representa um sistema de gases fermiônicos aprisionados por uma rede óptica quase periódica unidimensional e um potencial externo bidimensional. A seção 4 refere-se aos resultados obtidos como conseqüência da aproximação variacional para gaps sólitons iluminados em 3D. Na seção 5 são apresentadas as conclusões dos resultados obtidos e sugestões para trabalhos futuros, também incentivamos o leitor a realizar os cálculos variacionais para outras dimensões de aprisionamentos. 


\section{Uma breve abordagem sobre Sólitons e redes Ópticas.}

Um dos grandes precursores do estudo da propagação de ondas em meios aquáticos foi John Scott Russell em 1834, que ao observar a propagação de ondas aquáticas no canal de Eddinburgh-Glasgow notou que uma massa de água se propagava com velocidade constante sem perder a sua forma. Para uma definição mais precisa, o nome sóliton surgiu da expressão em inglês "solitary wave" (onda solitária). Uma simples ilustração de uma onda solitária propagando-se em um meio (canal) pode ser visualizada na Fig.1 abaixo. A onda está confinada pelas extremidades de uma caixa retangular de profundidadeH, comprimento-X, e largura-Y. Na Fig. 1, observa-se que a onda aquática se propaga ao longo da caixa retangular na direção X sem mudar a sua forma, tal comportamento também ocorre em guias dielétricos e surgem em decorrência do balanço entre efeitos não lineares e efeitos de dispersão do meio [27, 28].

Os sólitons não existem simplesmente em meio aquoso, o comportamento observado na Fig. 1 ocorre em outros meios como em fibras ópticas, sistemas biológicos e condensados de Bose-Einstein. Na física de estado sólido, o termo gap proibido é definido como regiões espectrais onde não existe a propagação de ondas; entretanto, quando a não linearidade permite a localização de ondas dentro de uma banda de gap linear, formam-se o que definimos de gaps sólitons iluminados [29]. No caso dos condensados de Bose-Einstein, as funções de ondas macroscópicas obedecem às soluções chamadas de equação de Gross-Pitaevskii, cuja não linearidade é um resultado das interações entre átomos. Dependendo da natureza de atração ou repulsão entre as partículas, a equação de Gross-Pitaevskii possui soluções do tipo sólitons iluminados para interações repulsiva (pico de intensidade localizada acima do fundo de uma onda contínua) ou escuros para interações atrativas entre partículas (caracterizado como uma queda de intensidade localizada abaixo do fundo de uma onda contínua) [26].

Experimentalmente a partir da sobreposição de lasers em três ou uma dimensão, criam-se potenciais que confi-

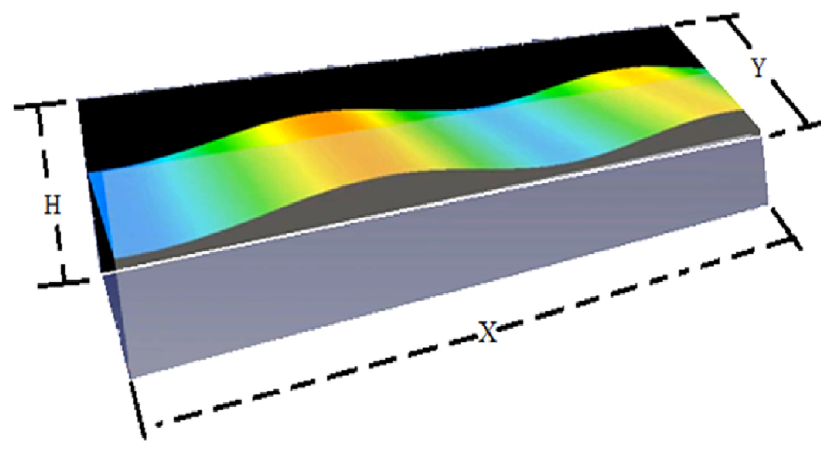

Figura 1: Caixa retangular de profundidade- $\mathrm{H}$, comprimento- $\mathrm{X}$, e largura-Y no qual uma onda solitária se propaga. nam os átomos [2]. Procedimento experimental similar também ocorre para produzir uma rede óptica unidimensional que se origina da intersecção de dois feixes de lasers contrapropagantes, que teoricamente pode ser escrito como,

$$
V(x)=\varepsilon_{1} \cos (2 x+\varphi)+\varepsilon_{2} \cos (\eta x)
$$

em que $\varepsilon_{1}$ e $\varepsilon_{2}$ são as amplitudes ou profundidade da rede óptica e podem ser ajustadas experimentalmente a partir da intensidade dos lasers. Experimentalmente, o parâmetro $\varphi$ na Equação (1) corresponde à fase relativa entre duas ondas, e $\eta$ é a razão entre os diferentes números de ondas de cada laser. Estudos considerando o mesmo potencial de aprisionamento, descrito na Equação (1) foram realizados experimentalmente envolvendo átomos bosônicos [30], e teoricamente, considerando átomos fermiônicos unidimensionais [26]. A partir das considerações citadas, adotaremos em nosso trabalho para a Equação (1), os seguintes valores: $\varepsilon_{1}=\varepsilon_{2}=\varepsilon ; \eta=1$ e $\varphi=1$, desta forma obtemos,

$$
V(x)=\varepsilon[\cos (2 x+1)+\cos (x)]
$$

O potencial descrito pela Equação 2 gera um padrão de interferência em que os átomos são aprisionados nos nodos e antinodos pela força de dipolo. A Fig.2 abaixo ilustra um exemplo do potencial de rede quase periódica que será utilizado em nosso trabalho obtido a partir da Equação (2). Outros valores para a amplitude de rede quase periódica também serão considerados na seção 4 .

O potencial descrito pela Equação (2) quando introduzido em uma equação hidrodinâmica para férmions ou também denominada de equação de densidade funcional, que é uma equação não linear devido à interação entre partículas, permite através de métodos variacionais obtermos soluções solitônicas. Tais soluções possuem a forma de sólitons iluminados e existem dentro de gaps proibidos do espectro da banda de gap linear, sendo então denominados de gaps sólitons iluminados [31]. Os

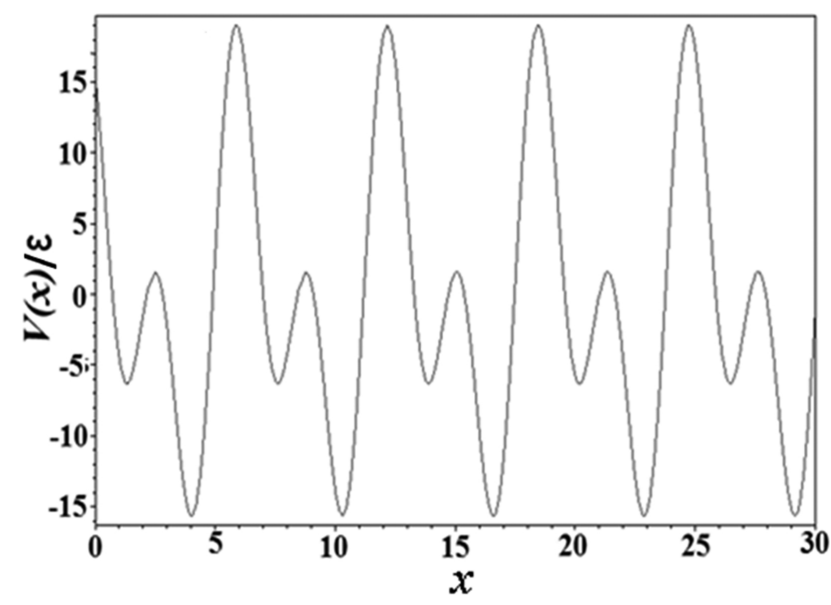

Figura 2: Exemplo de uma rede óptica unidimensional quase periódica criada pela intersecção de feixes de lasers contrapropagantes obtidas a partir da Equação (2). 
sólitons em condensados de Bose-Einstein surgem devido à presença da rede óptica e dos efeitos não lineares. Uma abordagem fundamental sobre sólitons em condensados de Bose-Einstein é encontrada na Ref.[32, 33]. Na física do estado sólido, as soluções da equação de equação de Schrödinger linear contendo um potencial criado pelos íons da rede e que atuam nos elétrons, produz uma dependência da energia das bandas de Bloch em função do quase momento, permitindo obtermos as estruturas de bandas da rede óptica e os seus respectivos gaps espectrais [29]. Fenômenos similares que ocorrem na física de estado sólido, ou seja, detecções de gaps espectrais também foram obtidas em estudos teóricos que envolvem gases fermiônicos e bosônicos aprisionados por redes ópticas [16, 24-26]. Evidências experimentais comprovaram que existe uma relação entre a física de átomos ultrafrios e física de estado sólido [34].

Apesar da Equação (2) fazer parte de vários estudos teóricos ou experimentais, potenciais na forma panqueca ou charuto também cumpre seu papel no processo de aprisionamento de átomos frios. Diante do exposto, nós utilizaremos o potencial total de aprisionamento de átomos escrito como,

$$
V(r)=\frac{1}{2} m \omega_{\perp}^{2}\left(y^{2}+z^{2}\right)+\varepsilon[\cos (2 x+1)+\cos (x)]
$$

em que o primeiro termo da Equação (3), ou seja, $V(y, z)=$ $(1 / 2) m \omega_{\perp}^{2}\left(y^{2}+z^{2}\right)$ refere-se ao potencial externo do tipo oscilador harmônico em $2 \mathrm{D}$ (na forma de charuto) que representa o potencial de aprisionamento magnético experimental, sendo ma massa atômica e $\omega_{\perp}$ é a freqüência do laser de aprisionamento transversal (ou freqüência do confinamento harmônico), e o segundo termo $V(x)=\varepsilon[\cos (2 x+1)+\cos (x)]$ trata-se do potencial da rede óptica quase periódica descrito pela Equação (2).

\section{Equação hidrodinâmica para férmions}

Uma boa introdução sobre a fundamentação teórica e obtenção da equação hidrodinâmica para férmions, também denominada de equação de densidade funcional, pode ser encontrada nos trabalhos publicados nas Ref.[16, 26]. Uma equação hidrodinâmica dependente do tempo que representa gases fermiônicos aprisionados por campos de luz, ou seja, lasers de aprisionamentos externos e potencial da rede quase periódica podem ser escritos como,

$$
\begin{aligned}
& i \hbar \frac{\partial \psi(r, t)}{\partial t}=\frac{\hbar^{2}}{2 m_{e f}}\left[-\nabla^{2} \psi(r, t)\right. \\
& \left.+\left(3 \pi^{2} / 2\right)^{2 / 3}|\psi(r, t)|^{4 / 3} \psi(r, t)\right]+V(r) \psi(r, t)(4)
\end{aligned}
$$

Na Equação (4), a função de onde $|\psi(r, t)|^{2} \equiv \rho_{3 D}$ é proporcional à densidade total de átomos, na qual é obtida levando em consideração os pares de Cooper $[16,26]$. O termo $\nabla^{2}=\partial^{2} / \partial x^{2}+\partial^{2} / \partial y^{2}+\partial^{2} / \partial z^{2}$ é o Laplaciano em 3D que representa a energia cinética. Ao contrário da equação de Gross-Pitaevskii que possui um termo de não linearidade cúbica repulsiva ou atrativa para bósons $|\psi(r, t)|^{2} \psi(r, t)[35]$, o parâmetro de ordem que descreve o superfluído fermiônico aprisionado por potenciais ópticos na Equação (4) é representado pelo termo não linear repulsivo $|\psi(r, t)|^{4 / 3} \psi(r, t)$. O último termo na Equação (4), ou seja, $V(r)$ trata-se do potencial na forma de charuto descrito pela Equação (3). A Equação (4) está sujeita à condição de normalização $\int\left|\psi(r, t)^{2}\right| d x d y d z=N$.

Para o presente caso em estudo, consideraremos a Equação 10 independente do tempo ao assumirmos $\psi(r, t)=e^{-i \mu_{D} t / \hbar} \varphi(r)$, onde $\mu_{D}$ é o potencial químico e $\phi(x)$ é uma função real sujeita à condição de normalização $\int \varphi^{2}(r) d r=1$. Além de reescrevermos a Equação (4) na forma estacionária, acrescentaremos a rede óptica quase periódica unidimensional e o potencial de confinamento do tipo oscilador harmônico bidimensional (Equação (3)). A partir de tais considerações, a Equação (4) passa a ser escrita como,

$$
\begin{aligned}
& \mu_{D} \varphi(r)=\frac{\hbar^{2}}{2 m_{e f}}\left[-\nabla^{2} \varphi(r)+\left(3 \pi^{2} / 2\right)^{2 / 3}|\varphi(r)|^{4 / 3} \varphi(r)\right]+ \\
& +\frac{1}{2} m \omega_{\perp}^{2}\left(y^{2}+z^{2}\right) \varphi(r)-\varepsilon[\cos (2 x+1)+\cos (x)] \varphi(r)
\end{aligned}
$$

De posse da Equação (5), podemos reduzi-la ao realizarmos o processo de escalonamento, para isso consideraremos:

$$
\begin{gathered}
\varphi(r) \equiv\left(\frac{2 \pi}{\lambda}\right)^{3 / 2} \sqrt{N} \tilde{\varphi}, \quad \mu_{D} \equiv \frac{\hbar^{2}}{m}\left(\frac{2 \pi}{\lambda}\right)^{2} \tilde{\mu}_{D} \quad \mathrm{e} \\
\chi \equiv \frac{\hbar^{2}}{m}\left(\frac{2 \pi}{\lambda}\right)^{2} \tilde{\chi}
\end{gathered}
$$

onde $\chi=x, y$ e $z$. A Equação (5) após reescalonamento passa ser escrita como

$$
\begin{aligned}
& \mu_{D} \varphi(r)=-\frac{1}{2}\left(\partial_{x}^{2}+\partial_{y}^{2}+\partial_{z}^{2}\right) \varphi(r)+g_{3 D} \\
& \times|\varphi(r)|^{4 / 3} \varphi(r)+\alpha\left(y^{2}+z^{2}\right) \varphi(r) \\
& -V_{0}[\cos (2 x+1)+\cos (x)] \varphi(r)
\end{aligned}
$$

Para a Equação (6) o termo de não linearidade é representado por $g_{3 D}=\frac{m_{e f}}{2 m}\left(\frac{2 \pi}{2}\right)^{3 / 2} N^{2 / 3}$, e para a função de onda temos: $\varphi(r) \equiv\left(\frac{2 \pi}{\lambda}\right)^{3 / 2} \sqrt{N} \tilde{\varphi}$. O termo $V_{0}=\varepsilon m_{e f}\left(\frac{\lambda}{2 \pi \hbar}\right)^{2}$ é proporcional à amplitude da rede onde $\lambda$ trata-se do comprimento de onda do laser de aprisionamento. Para simplificar os cálculos consideraremos: $m_{e f f} / m(\lambda / 2 \pi \hbar)^{2}=1$, a partir desta consideração $V_{0}=\varepsilon$. O termo $\alpha=(1 / 2) m \omega_{\perp}^{2}$ será considerado como uma constante.

A partir da Equação (6), obtemos a densidade lagrangiana de acordo com a Equação (7) abaixo

$$
\begin{aligned}
& L_{D}=+\mu_{D}|\varphi(r)|^{2}-\frac{1}{2}|\nabla \varphi(r)|^{2} \\
& +V_{0}[\cos (2 x+1)+\cos (x)]|\varphi(r)|^{2}+ \\
& -\alpha\left(y^{2}+z^{2}\right)|\varphi(r)|^{2}-\frac{3 g_{3 D}}{5}|\varphi(r)|^{10 / 3}
\end{aligned}
$$

A evolução da função de onda de um sistema de gases frios descritos pela Equação (7) é realizada através da minimização 
de $L_{D}$ por meio de um conjunto de funções tentativas (ansatz) na forma gaussiana. Conforme comprovado em vários estudos, as funções de ondas gaussianas são eficazes como soluções de equações que descrevem condensados bosônicos e fermiônicos [16, 24-26, 36-38]. Nós consideraremos como solução variacional para a Equação (7) a função tentativa variacional em $3 \mathrm{D}$ na forma gaussiana:

$$
\varphi(x, y, z)=\frac{\sqrt{N}}{\pi^{3 / 4} W V^{1 / 2}} \exp \left(-\frac{x^{2}+y^{2}}{2 W^{2}}-\frac{z^{2}}{2 V^{2}}\right)
$$

em que $N$ corresponde a norma, $W$ é a largura bidimensional e $V$ a largura axial do sóliton. Com o objetivo de encontrar as equações que fornecem a evolução dos parâmetros variacionais $W$ e $V$, substituímos a Equação (8) na Equação (7) para obtermos a lagrangiana efetiva através da densidade lagrangiana no espaço de coordenadas utilizando a relação,

$$
L_{e f}=\left\langle L_{D}\right\rangle=\int_{-\infty}^{\infty} \int_{-\infty}^{\infty} \int_{-\infty}^{\infty}\left[L_{D}\right] d x d y d z
$$

ou seja, a lagrangiana efetiva obtida a partir da Equação (7) é escrita como,

$$
\begin{aligned}
& L_{e f}=\int_{-\infty}^{\infty} \int_{-\infty}^{\infty} \int_{-\infty}^{\infty}\left[\mu_{D}|\varphi(x, y, z)|^{2}\right. \\
& -\frac{1}{2}|\nabla \varphi(x, y, z)|^{2}+V_{0}[\cos (2 x+1)+\cos (x)] \\
& \times|\varphi(x, y, z)|^{2}-\alpha\left(y^{2}+z^{2}\right)|\varphi(r)|^{2} \\
& \left.-\frac{3 g_{3 D}}{5}|\varphi(x, y, z)|\right] d x d y d z+\mu_{D} .
\end{aligned}
$$

Após integrarmos a Equação 10 em relação à $x, y$ e $z$ obtemos a lagrangiana efetiva em 3D para férmions aprisionados por uma rede óptica quase periódica unidimensional e na presença de um oscilador harmônico bidimensional, a qual é escrita em termos dos parâmetros da função de onda da gaussiana como,

$$
\begin{aligned}
& L_{e f}=\mu_{D}(N-1)-\frac{N}{2 W^{2}}-\frac{N}{4 V^{2}} \\
& -\frac{3^{5 / 2}}{5^{5 / 2} \pi} \frac{N^{5 / 3}}{W^{4 / 3} V^{2 / 3}} g_{3 D}++V_{0} N\left(0.54 e^{-W^{2}}+e^{-0.25 W^{2}}\right) \\
& -\frac{\alpha N V^{2}}{2}-\frac{\alpha N W^{2}}{2}
\end{aligned}
$$

O comportamento de $\mu_{\mathrm{D}}$, e a evolução dos parâmetros gaussianos $N, W$ e $V$ explícitos na Equação 111 são obtidas ao resolvermos as equações de Euler-Lagrange

$$
\frac{d}{d t}\left(\frac{\partial L_{e f}}{\partial \dot{q}_{j}}\right)-\frac{\partial L_{e f}}{\partial q_{j}}=0,
$$

em que adotamos como notação $q_{j}=\left\{\mu_{\mathrm{D}}, N, W, V\right\}$. A primeira equação variacional é obtida a partir da lagrangiana efetiva (Equação (11)), assim; $\partial L_{e f} / \partial \mu_{D}=0$ produz como esperado $N=1$, dessa forma $N=1$ será substituído nas outras equações variacionais abaixo. Desenvolvendo os cálculos $\partial L_{e f} / \partial V=\partial L_{e f} / \partial W=0$, teremos a relação entre a largura do sóliton $(W$ e $V)$ e $g_{3 D}$ conforme abaixo

$$
\frac{1}{2}+\frac{2}{3}\left(\frac{3}{5}\right)^{5 / 2} \frac{g_{3 D} V^{4 / 3}}{\pi W^{4 / 3}}=\alpha V^{4}
$$

$$
\begin{aligned}
& \frac{1}{W^{3}}+\frac{4}{3}\left(\frac{3}{5}\right)^{5 / 2} \frac{g_{3 D}}{\pi W^{7 / 3} V^{2 / 3}} \\
& +V_{0} W^{2}\left[1.08 e^{-W^{2}}+0.5 e^{-0.25 W^{2}}\right]=\alpha W^{2}
\end{aligned}
$$

A partir da equação $\partial L_{D, e f} / \partial N=0$, obtemos $\mu_{D}$ em função de $V \mathrm{e} W$, que resulta na expressão,

$$
\begin{aligned}
& \frac{1}{2 W^{2}}+\frac{1}{4 V^{2}}+\left(\frac{3}{5}\right)^{5 / 2} \frac{5 g_{3 D}}{3 \pi W^{4 / 3} V^{2 / 3}} \\
& +V_{0}\left(0.54 e^{-W^{2}}+e^{-0.25 W^{2}}\right)+\frac{\alpha V^{2}}{2} \\
& +\frac{\alpha W^{2}}{2}=\mu_{D}
\end{aligned}
$$

As soluções das Eqs. 13) e (14) produzem uma dependência de $g_{3 D}$ em função de $\mu_{D}$ para gaps sólitons iluminados em 3D. Os cálculos serão realizados considerando a amplitude da rede óptica quase periódica dentro de um intervalo $1 \leq V_{0} \leq 10$. Estando de posse da expressão da não linearidade em função do potencial químico, devemos determinar o valor de $V$ ou $W$ que minimiza esta expressão. Os dois sistemas de equações 13 e 14 são equivalentes; nesse caso, toda solução de qualquer um dos sistemas também é solução do outro. Uma solução de um sistema é uma seqüência de números (valores dos parâmetros variacionais) que satisfaz as equações simultâneas. Uma alternativa para resolvermos um sistema de equações lineares é utilizarmos o método de Gauss-Seidel (método de iteração), ou outros métodos abordados extensamente e disponíveis em livros-texto de física computacional e métodos numéricos.

\section{Resultados obtidos a partir da aproximação variacional}

Os resultados obtidos utilizando as aproximações variacionais para o presente caso em estudo estão expostos nas Figs.3, 4 e 5. Para todos os valores de $V_{0}$ considerados, o comportamento de $g_{3 D}$ versus $\mu_{3 D}$ em um gás fermiônico aprisionado por uma rede quase periódica e na presença de um potencial harmônico bidimensional são iguais.

A formação do gap, característica fundamental da estrutura de banda dos sólidos cristalinos, é nosso principal resultado no estudo envolvendo gases quânticos. As barras verticais na Fig.3 representam as primeiras bandas de Bloch que separam os gaps Para uma melhor compreensão desta possibilidade, o leitor deve lembrar que na física de estado sólido, uma equação de Schrödinger unidimensional linear com um potencial periódico do tipo $\varepsilon \cos (k x)$, permite o surgimento de um espectro com banda de gaps finitas separadas por distintas bandas de Bloch que possuem soluções de ondas lineares espacialmente quase periódicas [22]. Desta forma, processo similar também ocorre no estudo de gases quânticos, onde a não linearidade permite a localização de ondas dentro de uma banda de gap linear, formando gaps sólitons iluminados.

Em nosso trabalho, para obtermos os valores das bordas das bandas de Bloch, devemos considerar um regime linear, para isso nas Eqs. (13 e 14) admitiremos que $g_{3 D}=0$ e que o valor de amplitude da rede óptica seja $V_{0}=1$. A partir de tais considerações, as soluções variacionais das Eqs. (13) e (14) fornecem o valor para o potencial químico $\mu_{3 D}=1.0$ 

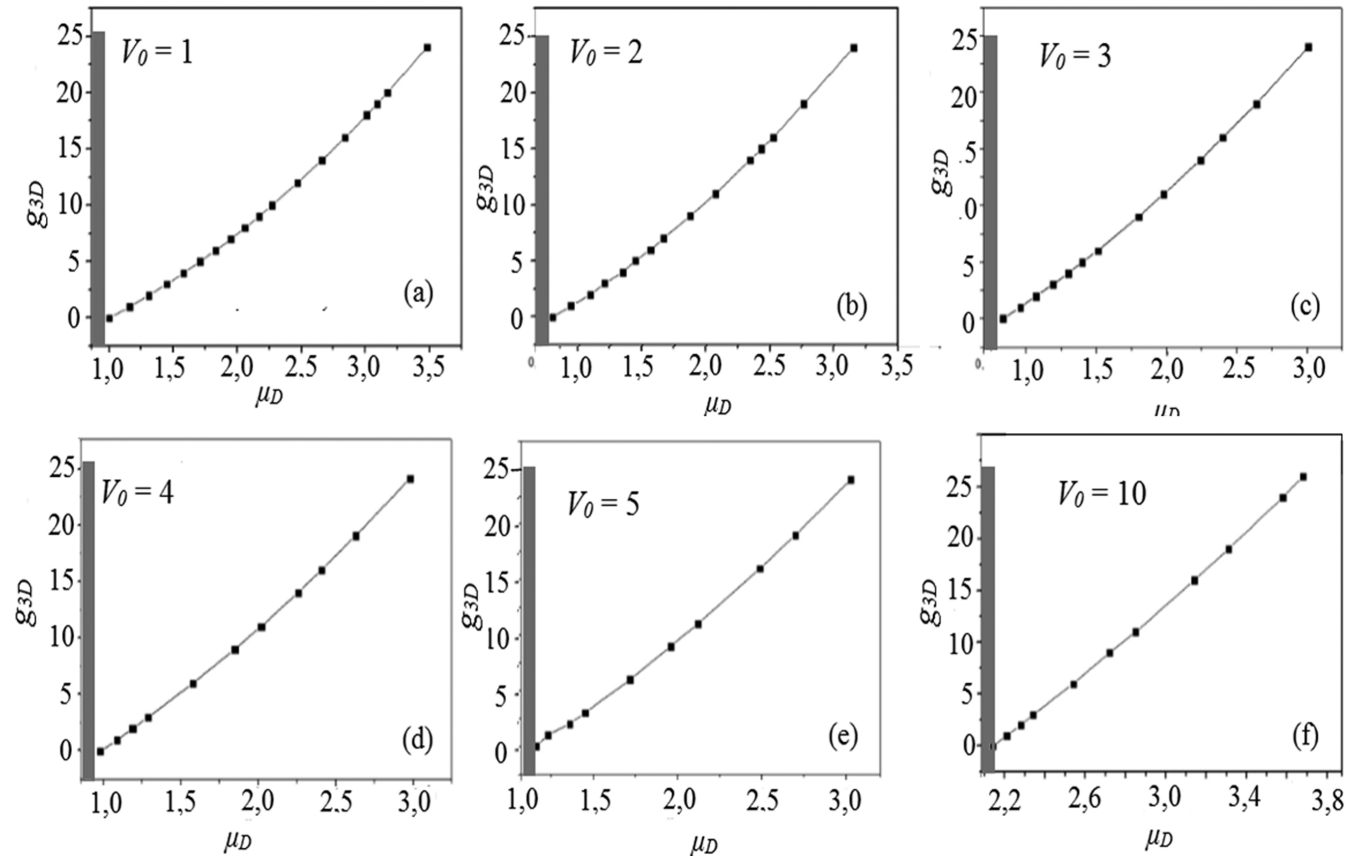

Figura 3: Resultados variacionais para $g_{3 D}$ versus $\mu_{\mathrm{D}}$ obtidos da primeira banda de gap para uma rede óptica quase periódica. Osvalores para os potenciais químicos foram obtidos considerando o potencial quase periódico " $V(x)=V_{0}[\cos (2 x+1)+\cos (x)]^{\prime \prime}$. A barra vertical representa a primeira banda de Bloch que separam os gaps

(Fig.3(a)), o qual coincide com o valor da primeira borda esquerda na primeira banda de gap. No segundo caso quando $V_{0}=2\left(g_{3 D}=0\right)$ obtivemos o seguinte valor para o potencial químico $\mu_{3 \mathrm{D}}=0.794$, que corresponde ao valor da primeira borda esquerda na primeira banda de gap (Fig3(b)). Para outros valores de amplitude de rede óptica quase periódica e regime linear $g_{3 D}=0$, obtemos $\mu_{3 \mathrm{D}}=0.83\left(V_{0}=3\right), \mu_{3 \mathrm{D}}=$ $0.99\left(V_{0}=4\right), \mu_{3 \mathrm{D}}=1.16\left(V_{0}=5\right), \mu_{3 \mathrm{D}}=1.36\left(V_{0}=6\right)$, $\mu_{3 \mathrm{D}}=1.55\left(V_{0}=7\right), \mu_{3 \mathrm{D}}=1.74\left(V_{0}=8\right), \mu_{3 \mathrm{D}}=1.95$ $\left(V_{0}=9\right)$ e $\mu_{3 \mathrm{D}}=2.14\left(V_{0}=10\right)$, no qual equivalem ao valor da respectiva borda para cada amplitude considerada. Por outro lado, para o regime não linear, no qual são adotados valores para a não linearidade $g_{3 D}>0$ é possível obtermos o comportamento da não linearidade em função do potencial químico para cada amplitude de rede óptica quase periódica

Famílias de sólitons iluminados, ou gaps sólitons iluminados, para gases fermiônicos são localizadas na primeira borda da banda de gap do potencial da rede quase periódica através das soluções das Eqs. (13) e 14 (Fig.3). De acordo com vários trabalhos publicados na literatura considerando outros tipos de redes ópticas, as soluções variacionais predizem famílias de gaps sólitons iluminados na primeira e através da segunda banda de gap, entretanto, tais soluções não sentem a presença das bandas de Bloch que separam as bandas de gap [16, 36, 39]. Diante do exposto, nosso estudo é realizado apenas para valores próximos à primeira borda da banda de gap.

Conforme comprovado em outros trabalhos [16, 24-26, 36, 39], gaps sólitons iluminados são localizadas em pequenos valores do coeficiente de não linearidade. Diante do exposto, para cada valor de amplitude de rede adotado, podemos estudar o comportamento dos vários tipos de gaps sólitons com distintos valores de $\mu_{\mathrm{D}}$ próximos das bordas das bandas de gap obtidos na Figura 3, para isso basta considerarmos a gaussiana que os representa (Equação (8) no qual possui como parâmetros a largura bidimensional $(W)$ e $V$ que se tratada largura axial. Os valores para as larguras $(W, V)$ são obtidas através da solução da Equação (13) para cada valor de amplitude selecionado. A partir das soluções das Equações 13. e 14 acopladas, obtém-se os valores para os potenciais químicos $\mu_{D}$ para cada respectivo valor de $V_{0}$. Considerando apenas a Fig.3(a) no qual a amplitude de rede óptica é $V_{0}=$ 1 e utilizando a Equação (8), observa-se a partir da Fig. 4 o comportamento de seis picos estáveis para gaps sólitons em 3D nas formas gaussianas obtidas próximas à borda da primeira banda de gap do espectro da Fig.3(a). Nos gráficos da Fig. 4 constam os valores de $g_{3 D}$ e $\mu_{D}$ para o caso de uma rede quase periódica com amplitude $V_{0}=1$.

Ao observarmos o comportamento dos gaps sólitons iluminados para um gás de Férmions em 3D na Fig.4, nota-se que, conforme aumenta o valor dos coeficientes de não linearidades, também aumentam os valores para os potenciais químicos e as larguras das gaussianas $V$ e $W$. Comportamento similar também foi observado em outros trabalhos teóricos envolvendo átomos fermiônicos aprisionados por redes ópticas simples ou duplamente periódicas [16, 24-26]. O aumento de $g_{3 D}$ versus $\mu_{D}$ pode ser observado à medida que nos afastamos da borda da primeira banda de gap (Veja Fig.3(a)), ou seja, as famílias de gaps sólitons iluminados em condensados repulsivos originam do fundo da banda de gap. Isto é, quando a não linearidade é fraca, o número de átomos é pequeno e estados de gaps sólitons iluminados surgem próximos das bordas inferiores das bandas de gap linear. Por outro lado, devido ao aumento do potencial químico, também haverá uma alteração no número de átomos no gap.

Os resultados apresentados na Fig.3 para os valores dos potenciais químicos obtidos ao variarmos a amplitude da rede 

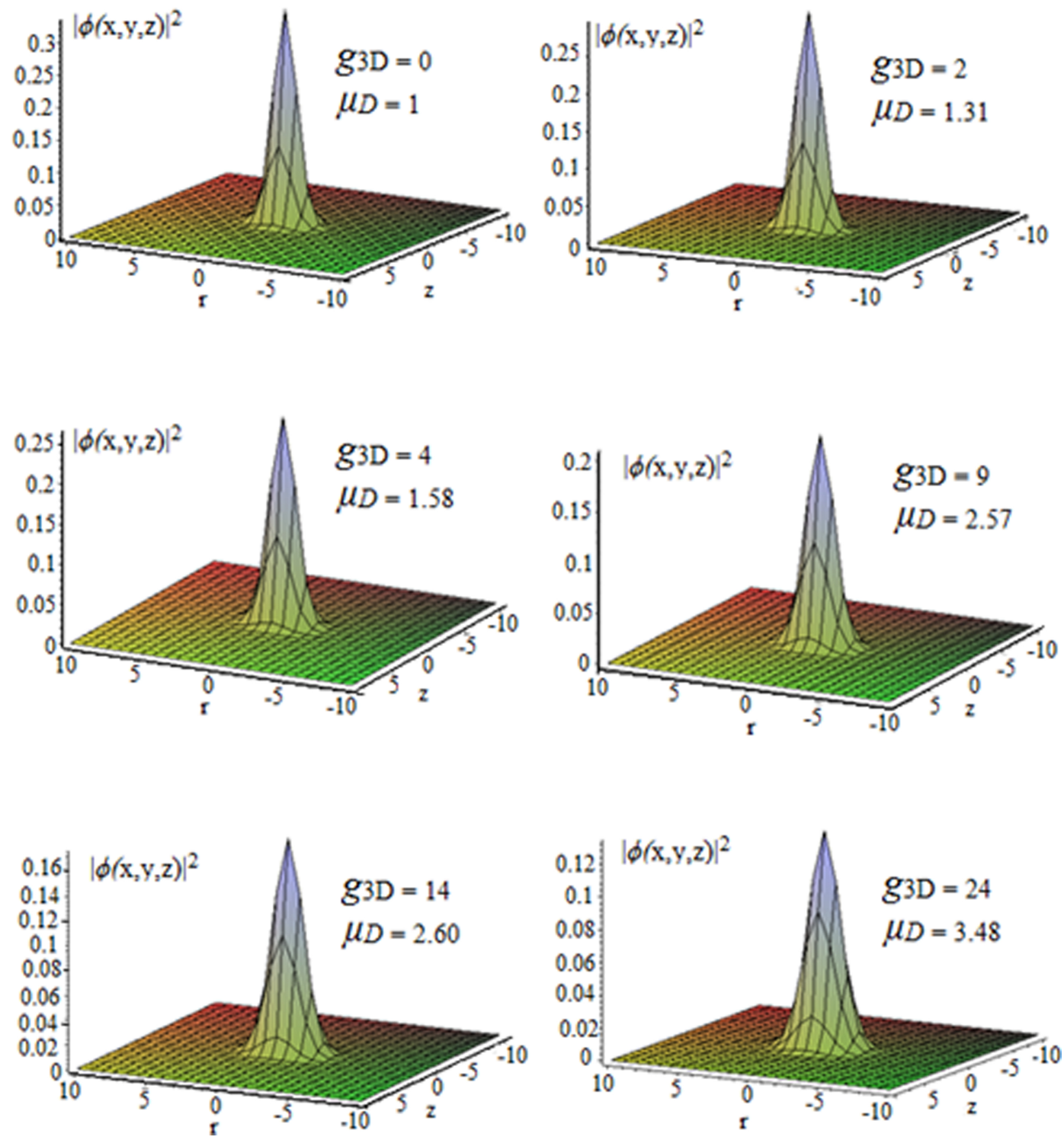

Figura 4: Formas típicas de gaps sólitons iluminados para um gás de férmions em 3D. Exemplo de seis picos estáveis para gaps sólitons na forma gaussiana (Equação (8)) obtidos próximos à borda da primeira banda de gap do espectro da Fig. 3(a). Nos gráficos constam os valores de $g_{3 D}$ e $\mu_{D}$ para o caso de uma rede quase periódica: Fig.3(a) para $V_{0}=\varepsilon=1$. Nesta figura nós consideramos $r^{2}=x^{2}+y^{2}$.

quase periódica podem ser interpretados através da Fig.5 que representa $\mu_{3 D}$ versus $V_{0}$, onde os valores adotados para a amplitude da rede quase periódica estão dentro do intervalo $1 \leq V_{0} \leq 10$. Neste intervalo adotado, o comportamento de $g_{3 D}$ versus amplitude da rede quase periódica unidimensional $\left(V_{0}\right)$ que aprisionam o gás fermiônico comporta-se da mesma forma que gases bosônicos aprisionados por uma rede simples unidimensional [15, 24] e gases fermiônicos aprisionados por redes ópticas quase periódicas unidimensional sem a presença do potencial externo [16, 26].

Durante os últimos anos na tentativa de compreender os processos de interações entre átomos frios aprisionados por potenciais ópticos, surgiram estudos teóricos envolvendo o número de átomos em função do potencial químico $[16,36$, 40-42], potencial químico em função da amplitude da rede duplamente periódica ou em função de uma rede óptica simples [16, 24-26, 40, 41]. Porém, trabalhos teóricos, experimentais ou teórico-experimentais que estudam o comportamento do coeficiente de não linearidade em função do potencial químico para os casos de redes ópticas quase periódicas e potenciais externos do tipo charuto são escassos na literatura. É interessante ressaltarmos que os valores adotados em nosso trabalho como os parâmetros de ajuste da Equação (3), amplitude ou profundidade da rede duplamente periódica $V_{0}=5$, são os mesmos adotados em trabalhos numéricos [40].

Os resultados apresentados neste trabalho fornecem importantes contribuições para o estudo da aprendizagem de átomos frios aprisionados por campos de luz, uma vez quese tratade um trabalho inédito no qual se considera um gás de férmions descrito por uma equação hidrodinâmica em 3D contendo um potencial harmônico bidimensional e uma rede óptica quase periódica unidimensional que aprisionam os átomos. Como exercício, o leitor pode transformar a Equação (5) tridimensional em uma equação unidimensional utilizando a 


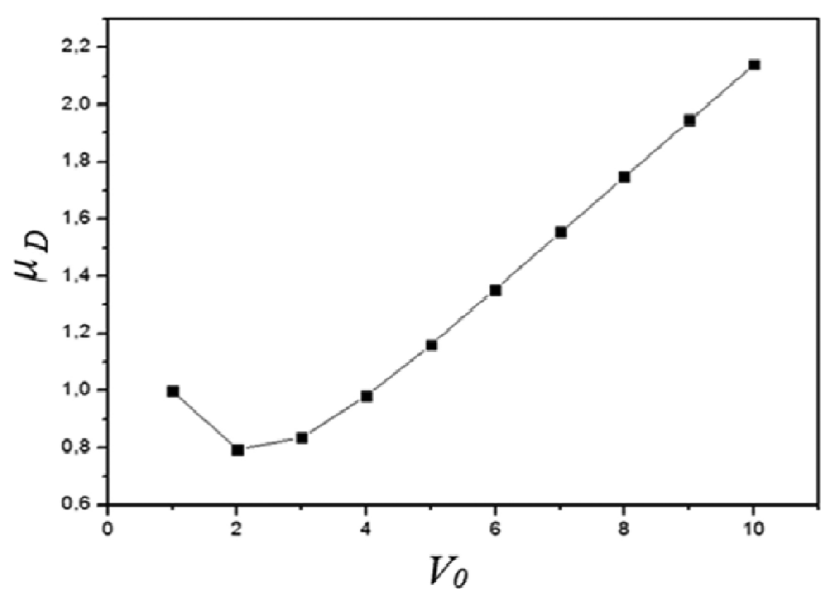

Figura 5: Comportamento de $\mu_{3 D}$ versus $V_{0}$ para um regime linear $\left(g_{3 D}=0\right)$. Consideramos o intervalo: $1 \leq V_{0} \leq 10$.

relação $\varphi(x, y, z)=\phi(x) \exp \left(-\left(y^{2}+z^{2}\right) / 2 a_{h o}^{2}\right)$, e obter sua solução através de uma função gaussiana. Maiores detalhes dos cálculos a serem realizados podem ser encontrados nas Ref. [24-26].

\section{Conclusão}

Neste artigo, nós demonstramos que o método variacional revela-se eficaz em descrever e prever famílias de gaps sólitons iluminados provenientes de gases fermiônicos aprisionadas por um potencial unidimensional de uma rede óptica quase periódica e na presença de um potencial da armadilha magneto óptica na forma de charuto. Através da aproximação variacional nós demonstramos a possibilidade de que o coeficiente de não linearidade para férmions atuando em combinação com o potencial de rede óptica quase periódica permite o surgimento de gaps sólitons iluminados em 3D.

A existência e o comportamento do coeficiente de não linearidade repulsiva versus potencial químico, além de ser observado em estudos teóricos envolvendo a equação de GrossPitaevskii em 1D, 2D e 3D envolvendo vários de tipos de redes ópticas e na presença ou ausência de potenciais externos, também é possível ao utilizarmos a configuração proposta em nosso trabalho.

Os resultados apresentados neste artigo abrem portas para a realização de futuros trabalhos, como o estudo teórico de gaps sólitons iluminados em 1D e 2D confinados por potenciais de redes ópticas quase periódica unidimensionais para um gás de férmions degenerados; estabilidade de gaps sólitons devido ao movimento da rede óptica quase periódica, ou seja, regime estável para instável e efeitos colisionais entre sólitons.

Os cálculos realizados podem ser reproduzidos por alunos de graduação ou pós-graduação de cursos de ciências exatas, para isso basta seguir os detalhes matemáticos e considerações físicas apresentadas nas subseções anteriores ou em outros trabalhos conforme as referências citadas. O comportamento dos gaps sólitons iluminados obtidos no presente caso em estudos são realizados considerando uma equação hidrodinâmica em $3 \mathrm{D}$. Incentivamos ao leitor realizar os cálculos variacionais transformando a Equação (5) tridimensional em uma equação unidimensional, e obter sua solução através de uma função gaussiana.

\section{Agradecimentos}

Gostaríamos de agradecer ao Prof. Dr. Sadhan K. Adhikari (IFT/UNESP) pelos valiosos ensinamentos na aprendizagem de métodos variacionais.

\section{Referências}

[1] M.H. Anderson, J.R. Ensher, M.R. Matthews, C.E. Wieman e E.A. Cornell, Science 269, 198 (1995).

[2] C.A. Regal, M. Greiner, D.S. Jin, Phys. Rev. Lett. 92, 040403 (2004).

[3] M. Bartenstein, A. Altmeyer, S. Riedl, S. Jochim, C. Chin, J. Hecker Denschlag e R. Grimm. Phys. Rev. Lett.92, 120401 (2004).

[4] D. Jaksch, V. Venturi, J.I. Cirac, C.J.Williams e P. Zoller, Phys. Rev. Lett. 89, 040402 (2002).

[5] M.P.A. Fisher, P.B. Weichman, G. Grinstein e D.S. Fisher, Phys. Rev. B. 40, 546570 (1989).

[6] M. Grainer, O. Mandel e I. Bloch, Nature 415, 39 (2002).

[7] O. Morsch, J.H. Müller, M. Cristiani, D. Ciampini e E. Arimondo, Phys. Rev. Lett. 87, 140402 (2001)

[8] I. Carusotto, L. Pitaevskii, S. Stringari, G. Modugno e M. Inguscio, Phys. Rev. Lett. 95, 093202 (2005).

[9] G. Dong, J. Zhu, W. Zhang e B.A. Malomed, Phys. Rev. Lett. 110, 250401 (2013).

[10] B. Eiermann, T. Anker, M. Albiez, M. Taglieber, P. Treutlein, K.P. Marzlin e M.K. Oberthaler, Phys. Rev. Lett. 92, 230401 (2004).

[11] L. Khaykovich, F. Schreck, G. Ferrari, T. Bourdel, J. Cubizolles, L.D. Carr, Y. Castin e C. Salomon, Science 296, 1290 (2002).

[12] S.L. Cornish, S.T. Thompson e C.E. Wieman, Phys. Rev. Lett. 96, 170401 (2006).

[13] J.V. Porto, S. Rolston, B.L. Tolra, C.J. Williams and W.D. Phillips Phil. Trans. Math. Phys. Eng. Sci. 361, $1471(2003)$

[14] K.G.H. Vollbrecht, E. Solano e J.I. Cirac, Phys. Rev. Lett.93, 220502 (2004).

[15] T. Mayteevarunyoo e B.A. Malomed, Phys. Rev. A. 74, 033616 (2006).

[16] B.A. Malomed, V.A. Nascimento e S.K. Adhikari, Mathematics and Computers in Simulation 80, 648 (2009).

[17] K. Huang, Statistical Mechanics (John Wileyand Sons, New York,1988).

[18] S.R.A. Salinas, Introdução à Física Estatística (Editora Universidade de São Paulo, São Paulo, 2013) $2^{\mathrm{a}}$ ed.

[19] S.R. Dahmen, Rev. Bras. Ens Fís. 27, 271 (2005).

[20] S.R. Dahmen, Rev. Bras. Ens Fís. 27, 283 (2005)

[21] N.D. Gomes, M.A. Caracanhas e V.S. Bagnato, Rev. Bras. Ens. Fís. 36, 1311 (2014).

[22] MA. Reis e S.A. Vitiello, Rev. Bras Ens. Fís28 45(2006)

[23] JCBAraujo, GR.P. Borges e ED Filho, Rev. Bras Ens Fís28, 41(2006).

[24] V.A. Nascimento, Rev. Bras. Ens. Fís. 33, 2305 (2011).

[25] V.A. Nascimento, C.L. Silva, A.V.D. Lanoa, V.A. Nascimento, A.F. Silva e P.C. Cassino, Rev. Bras. Ens. Fís.36, 01(2014).

[26] V.A. Nascimento, C.L. Silva, A.F. Silva, R.C.A. Guimarães e E.S.P. Melo, Rev. Bras. Ens. Fís. 38, e4301(2016).

[27] A.C.C. Fabio e J.P. Zubelli, Matemática Universitária 10, 41 (2001). 
[28] W. Galléas, L.H. Ymai, P.L. Natti, E.R. Takano Natti, Rev. Bras. Ens. Fís. 25, 294(2003).

[29] C. Kittel, Introduction to Solid State Physics (Willey \& Sons, New York, 2005) $8^{\mathrm{a}} \mathrm{ed}$.

[30] R.B. Diener, G.A. Georgakis, J. Zhong, M. Raizene Q. Niu, Phys. Rev. A 64, 033416 (2001).

[31] B.B. Baizakov, B.A. Malomed e M. Salerno, Phys. Rev. E. 74, 066615 (2006).

[32] B.A. Malomed, Soliton Management in Periodic Systems (Springer Science+Busines Media, Berlim, 2006).

[33] C.J. Pethick and H. Smith, Bose-Einstein condensation in Dilute Gases (Cambridge University Press, Cambridge, 2002).

[34] D. Greif, M.F. Parsons, A. Mazurenko, C.S. Chiu, S. Blatt, F. Huber, G. Ji e M. Greiner, Science 351, 953 (2016).

[35] C.J. Pethick and H. Smith, Bose-Einsten in diluite Gases (Cambridge University Press, Cambridge, 2002)

[36] C.T. Giner, R. Cipolatti e T.C.H. Liew, Eur. Phys. J. D. 67, 143 (2013).

[37] V.M.P. Garcia, H. Michinel, J.I. Cirac, M. Lewenstein e P. Zoller, Phys. Rev. Lett. 77, 5320 (1996).

[38] V.M. Pérez Garcia, H. Michinel, J.I. Cirac, M. Lewenstein and P. Zoller, Phys. Rev. A. 56, 1424 (1997).

[39] S.K. Adhikari e B. A. Malomed, Europhys. Lett. 79, 50003 (2007).

[40] P.J.Y. Louis, E.A. Ostrovskaya, C.M. Savage e Y.S. Kivshar, Phys. Rev. A. 67, 013602 (2003).

[41] P.J.Y. Louis, Elena A. Ostrovskaya e Y.S. Kivshar, Phys. Rev. A. 71, 023612 (2005).

[42] A. Gubeskys, B.A. Malomed e I.M. Merhasin, Etud. Appl. Math. 115, 255 (2005). 